\title{
Inspection of refractive $x$-ray lenses using high-resolution differential phase contrast imaging with a microfocus $x$-ray source
}

\author{
Martin Engelhardt ${ }^{\mathrm{a})}$ \\ Siemens AG, Corporate Technology, 81739 Munich, Germany and Physik Department, Technische Universität \\ München, 85748 Garching, Germany \\ Joachim Baumann and Manfred Schuster \\ Siemens AG, Corporate Technology, 81739 Munich, Germany \\ Christian Kottler, Franz Pfeiffer, Oliver Bunk, and Christian David \\ Paul Scherrer Institut, 5232 Villigen PSI, Switzerland
}

(Received 25 May 2007; accepted 28 August 2007; published online 26 September 2007)

A refractive $\mathrm{x}$-ray lens was characterized using a magnifying cone beam setup for differential phase contrast imaging in combination with a microfocus $\mathrm{x}$-ray tube. Thereby, the differential and the total phase shift of $\mathrm{x}$ rays transmitted through the lens were determined. Lens aberrations have been characterized based on these refractive properties. (c) 2007 American Institute of Physics.

[DOI: $10.1063 / 1.2786273$ ]

\section{INTRODUCTION}

Even though hard x rays are weakly refracted, in 1996, a successful application of a refractive $\mathrm{x}$-ray lens was presented $^{1}$ and opened a new field of $\mathrm{x}$-ray optics. In order to compensate the weak refraction, these lenses have a strong curvature. Furthermore, they may be stacked behind each other in large numbers. This arrangement is called compound refractive lens (CRL).

Meanwhile, refractive x-ray lenses play an important role in x-ray optics, especially at synchrotron beamlines. In full-field microscopy, ${ }^{2-5}$ the transmission image of an object is magnified onto the detector using X-ray lenses. Another application is scanning microscopy, ${ }^{2,3,5}$ where the lenses focus the incident beam onto the sample, and a secondary $\mathrm{x}$-ray analytical signal such as the fluorescence, the absorption, or a diffraction pattern is recorded. Finally, x-ray lenses are used for beam conditioning ${ }^{2}$ at synchrotron radiation sources, e.g., for focusing or collimation.

The functional principle of an $\mathrm{x}$-ray lens is outlined in Fig. 1. For visible light, the real part of the refractive index of matter is larger than the corresponding index of vacuum. For $\mathrm{x}$ rays, this relationship is vice versa. Therefore, a converging x-ray lens is concave shaped. In the following, a coordinate system aligned to the lens axis, with $x$ and $y$ being transversal axes and $z$ the propagation direction, is used. The $\mathrm{x}$ rays passing through the lens are deflected in the $x$ direction by an angle of

$$
\alpha_{\text {lens }}=\frac{\lambda}{2 \pi} \frac{\partial \phi_{\text {lens }}}{\partial x_{\text {lens }}},
$$

where $\partial \phi_{\text {lens }} / \partial x_{\text {lens }}$ is the differential phase shift in the $x$ direction of the $\mathrm{x}$ rays transmitted through the lens. In analogy, the refraction angle in any direction in the $x-y$ plane can be described. As shown in Fig. 1, in order that the lens focuses

\footnotetext{
${ }^{a)}$ Electronic mail: martin.engelhardt.ext@siemens.com
}

the $\mathrm{x}$ rays onto the focal point, the beam deflection angle $\alpha_{\text {lens }}$ and thus $\partial \phi_{\text {lens }} / \partial x_{\text {lens }}$ must be linearly proportional to $x_{\text {lens }}$, which means that the lens must have a parabolic shape. For visible light, this can often be approximated by a spherical shape. However, in the case of a refractive $\mathrm{x}$-ray lens, this would lead to unacceptable spherical aberrations because of the strong curvature of the lens.

For a single lens, the focal distance $f$ can be calculated from the radius of curvature $R$ of the lens at the apex of the parabolas by ${ }^{3}$

$$
f=\frac{R}{2 \delta},
$$

where $\delta$ is the refractive index decrement of the lens material.

Currently, quality validation of refractive $\mathrm{x}$-ray lenses is often accomplished at synchrotron beamlines. This can be done by magnifying an $\mathrm{x}$-ray transmission image of a test object onto the detector using a compound refractive lens. ${ }^{3-5}$ The spatial resolution and the distortions of the transmission image are a measure for the performance of the compound refractive x-ray lens. However, in order to improve manufacturing processes, a precise measurement of the refractive properties of a single x-ray lens would be valuable. Furthermore, it would be of advantage to determine the properties of the lens at its production facilities without the need of scheduling synchrotron beam time. For that reason, a laboratory setup for the precise determination of the refractive properties of $\mathrm{x}$-ray lenses was developed.

\section{GRATING BASED INTERFEROMETER}

An outline of the setup is shown in Fig. 2. It is based on $\mathrm{x}$-ray grating interferometry. ${ }^{6-9}$ The phase grating $\mathrm{g}_{1}$, with lines showing negligible $\mathrm{x}$-ray attenuation but a phase shift of $\pi$, serves as a beam splitter. It divides the incoming beam mainly into the -1 st and +1 st diffraction orders. Downstream 


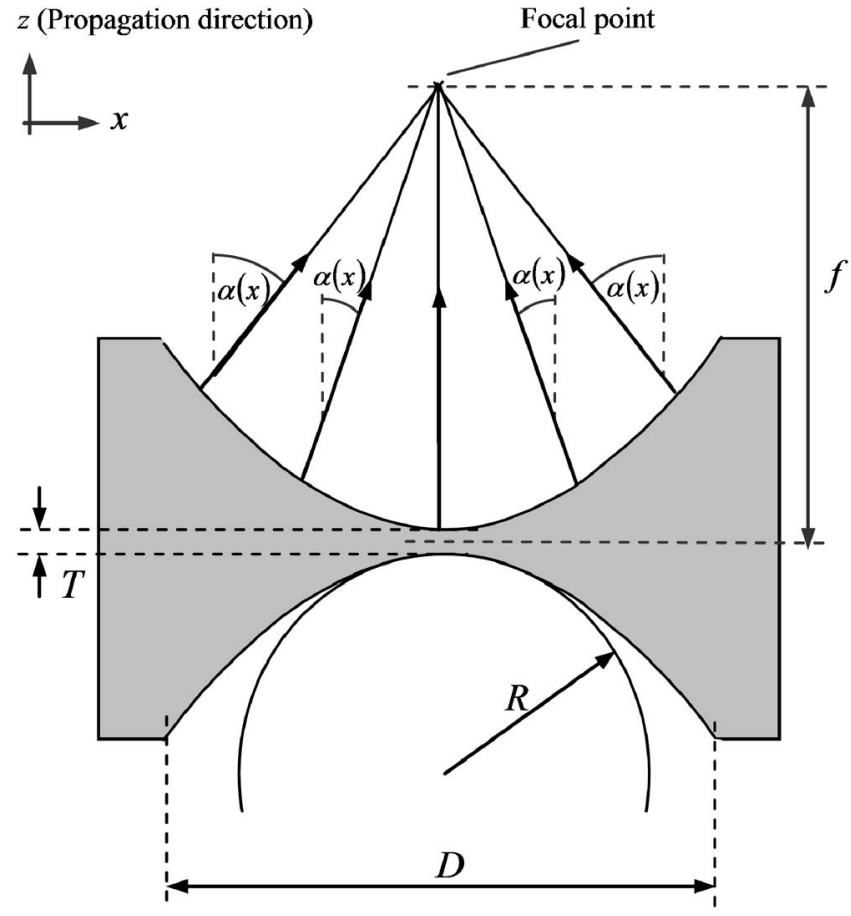

FIG. 1. X-ray beam path through a converging x-ray lens. The focal length of the lens is denoted by $f$ and the radius of curvature at the center of the parabolas by $R$.

of $\mathrm{g}_{1}$, the diffracted beams interfere and form a periodic interference pattern in planes parallel to $\mathrm{g}_{1}$. As described by the fractional Talbot effect, ${ }^{10}$ this pattern exhibits a maximum modulation at the following distances:

$$
d_{m}=m \frac{p_{1}^{2}}{8 \lambda}
$$

for a parallel beam setup, ${ }^{8}$ where $\lambda$ is the wavelength, $p_{1}$ is the period of $\mathrm{g}_{1}$, and $m$ is an odd integer, which corresponds to the fractional Talbot distance order. For a cone beam setup, these distances are ${ }^{11}$

$$
d_{m}^{*}=\frac{l}{l-d_{m}} d_{m} \text {. }
$$

In order to obtain a reasonable modulation of the interference pattern, the size of the source $\xi$ must be chosen to be small enough so that the transversal coherence length $\Lambda_{c}=\lambda l / \xi$ at the plane of $\mathrm{g}_{1}$ is larger than the separation

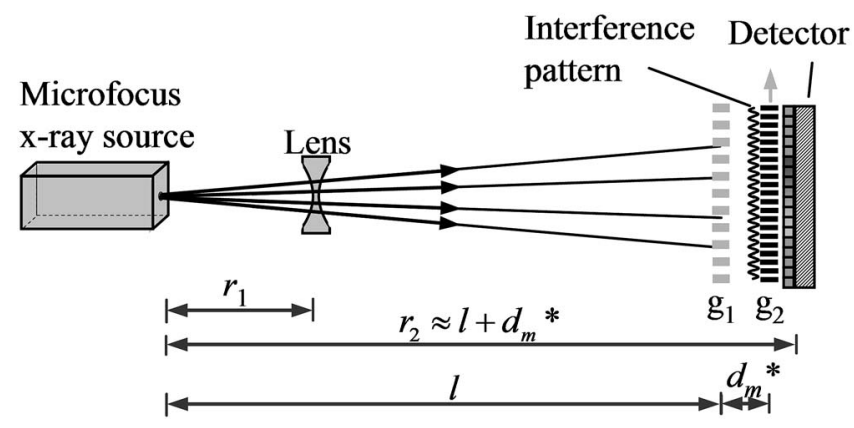

FIG. 2. Setup for differential phase contrast imagings using projection magnification. The setup is based on a phase grating and an absorption grating, which are denoted by $\mathrm{g}_{1}$ and $\mathrm{g}_{2}$, respectively. $h_{m}=m p_{1} / 4$ of the interfering beams. Perturbations of the incident wavefront due to an object in the beam path lead to a modification of the diffraction angles of ${ }^{7}$

$$
\alpha_{\mathrm{PG}}=\frac{\lambda}{2 \pi} \frac{\partial \phi_{\mathrm{PG}}}{\partial x_{\mathrm{PG}}},
$$

where $\partial \phi_{\mathrm{PG}} / \partial x_{\mathrm{PG}}$ is the differential phase shift at the phase grating plane. Thereby, the interference fringes are shifted by

$$
s=d_{m}{ }^{*} \alpha_{\mathrm{PG}} .
$$

This means that a determination of the fringe shift $s$ yields the differential phase shift $\partial \phi_{\mathrm{PG}} / \partial x_{\mathrm{PG}}$. The position of the fringes of the interference pattern is determined by scanning it $^{8}$ with the absorption grating $\mathrm{g}_{2}$, which consists of strongly absorbing lines with a period that matches $p_{2}{ }^{*}$. In this process, several images are acquired with a different relative position of $\mathrm{g}_{1}$ and $\mathrm{g}_{2}$. As outlined in Ref. $9, \partial \phi_{\mathrm{PG}} / \partial x_{\mathrm{PG}}$ is related to $\partial \phi_{\text {lens }} / \partial x_{\text {lens }}$ by

$$
\frac{\partial \phi_{\mathrm{PG}}}{\partial x_{\mathrm{PG}}}=\frac{r_{1}}{l} \frac{\partial \phi_{\text {lens }}}{\partial x_{\text {lens }}} .
$$

According to Eqs. (5)-(7), from the pattern shift $s$, the differential phase shift caused by the lens $\partial \phi_{\text {lens }} / \partial x_{\text {lens }}$ can be obtained, and so can the beam deflection angle of the lens [Eq. (1)], which is an ideal measure for the focusing performance of the lens. The method is also applicable for a polychromatic energy spectrum. The interference fringes show a certain modulation $^{8}$ for a relatively large photon energy spectrum. A simple approach to account for a polychromatic energy spectrum is to assign an effective energy. ${ }^{7}$

The source-detector distance of the setup was $r_{2}$ $=1770 \mathrm{~mm}$. The silicon phase grating $\mathrm{g}_{1}$ had a period $p_{1}$ of $3.82 \mu \mathrm{m}$. The lines thereof were $23 \mu \mathrm{m}$ high, which is optimal for $17.5 \mathrm{keV} x$ rays, as, in this case, they yield a phase shift of $\pi$. The absorption grating $\mathrm{g}_{2}$ had lines of $24 \mu \mathrm{m}$ gold with a period $p_{2}$ of $2 \mu \mathrm{m}$. The intergrating distance was set to the third fractional Talbot distance $\left(d_{3}{ }^{*}=81 \mathrm{~mm}\right.$ for $17.5 \mathrm{keV}$ photon energy). As a detector system, a charged coupled device (CCD) camera was combined with a Hamamatsu CsI scintillator. The spatial resolution, limited by the scintillator, is approximately $100 \mu \mathrm{m}$, whereas a minimum pixel size of $50 \mu \mathrm{m}$ is possible. A Hamamatsu microfocus tube with a $\mathrm{W}$ target and a focal spot size of approximately $8 \mu \mathrm{m}$ was used as an $\mathrm{x}$-ray source. It was operated at $50 \mathrm{kV}$ and $100 \mu \mathrm{A}$.

\section{LENS TEST}

A concave parabolic Be $\mathrm{x}$-ray lens, which was known to be defective, was investigated. It was made by Technische Universität Aachen, Germany from polycrystalline beryllium using a pressing tool. Thereby, two convex paraboloids with rotational symmetry facing each other were simultaneously pressed into a beryllium plate from both sides. ${ }^{3,4}$ Normally, the paraboloids are pressed deep into the beryllium such that a distance $T$ between the apices of the paraboloids of only $30 \mu \mathrm{m}$ remains. In this case, the diameter $D$ of the impressions will be approximately $880 \mu \mathrm{m}$. However, for the defective lens, $T$ was approximately $350 \mu \mathrm{m}$ and $D$ was ap- 
(a)

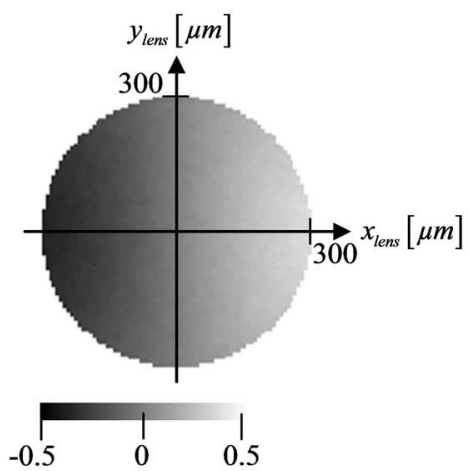

(b)

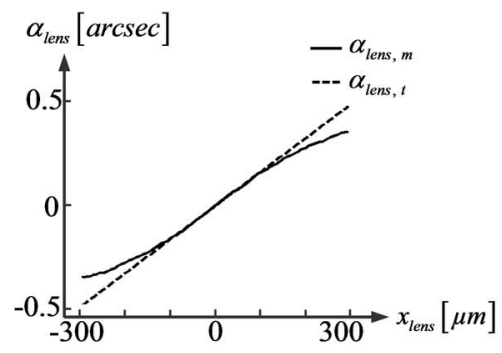

(c)

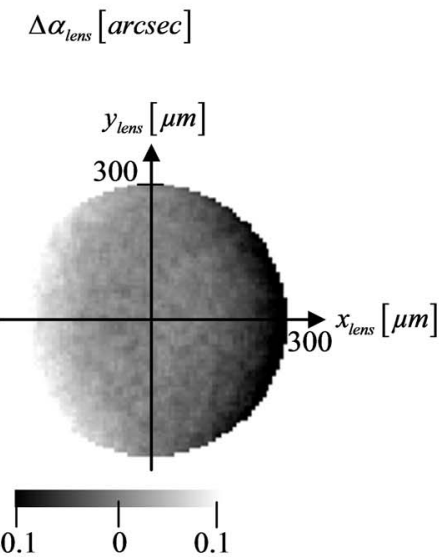

(d)

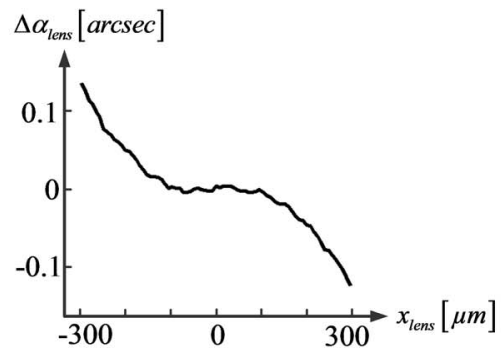

FIG. 3. Characterization of the refractive properties of a converging $\mathrm{x}$-ray lens made of beryllium based on the beam deflection angle. The measured beam deflection angle $\alpha_{\text {lens }, m}$ in the $x$ direction is presented in (a). In (b), a comparison of a line plot along the $x$ axis of $\alpha_{\text {lens }, m}$ (solid line) to its linear target behavior $\alpha_{\text {lens,t }}$ (dashed line) is shown. Lens errors can be characterized based on the difference $\Delta \alpha_{\text {lens }}$ between these two functions, which is illustrated as spatially resolved in (c) and as a line plot along the $x$ axis in (d). proximately $750 \mu \mathrm{m}$. Using the test setup described, the spatially resolved beam deflection angle of $\mathrm{x}$ rays transmitted by the lens was determined. By the aid of a laser, the rotation axis of the lens was aligned with the optical axis of the setup (centered and parallelized). This was done in order to avoid aberrations, which would otherwise occur for an intact x-ray lens due to the strong curvature of the lens.

A projection magnification of $M=r_{1} / r_{2}=6.3$ was chosen, yielding an effective spatial resolution of approximately $16 \mu \mathrm{m}$. The beam deflection angle $\alpha_{\text {lens, } m}$ at the lens in the direction of an arbitrary diametric axis $x$ was derived from a measurement of the differential phase shift. It is displayed as a function of $x$ and $y$ in Fig. 3(a). The beam deflection angle $\alpha_{\text {lens, } m}$ must be proportional to $x_{\text {lens }}$ in order that the incident $\mathrm{x}$ rays are focused onto the focal spot. In Fig. 3(b), a line plot along the $x$ axis of $\alpha_{\text {lens, } m}$ is compared to its linear target

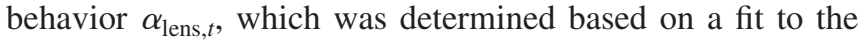
central part of the measurement data. The difference $\Delta \alpha_{\text {lens }}$

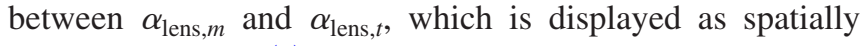
resolved in Fig. 3(c) and as a line plot along the $x$ axis in Fig. 3(d), can be used to characterize lens errors.

The images, shown in Figs. 3(a) and 3(c), are subject to noise, which was characterized based on further images, acquired with the same settings but without the lens in the beam path. The standard deviation of the measured beam deflection angle $\sigma_{\alpha_{\text {lens }, m}}$ for many different pixels, for which the beam deflection angle has the same target value of zero, was determined to be $\sigma_{\alpha_{\text {lens }, m}} \approx 0.008$ arc sec for the pixel size of $50 \mu \mathrm{m}$. For each point of the line plots in Figs. 3(b) and $3(\mathrm{~d})$, nine data points were averaged in the $y$ direction, which reduces the noise to $\sigma_{\alpha_{\text {lens }, m}} \approx 0.003$ arc sec.
The data can be interpreted using ray optics, whereby diffraction effects of the lens aperture are neglected. For example, the target value $\alpha_{\text {lens, } t}$ of 0.16 arc sec at $x=100 \mu \mathrm{m}$ yields a focal distance $f$ of $129 \mathrm{~m}$. This result is expected from the target lens curvature of $R=200 \mu \mathrm{m}$ for an effective energy of $E_{\text {eff }}=21 \mathrm{keV}$ [Eq. (2)]. The maximum deviation $\Delta \alpha_{\text {lens }}$ of the measured value from the target value for $|x| \leqslant 100 \mu \mathrm{m}$ is $\pm 0.004 \mathrm{arc} \mathrm{sec}$, which is of the order of the noise level of $\sigma_{\alpha_{\text {lens }, m}} \approx 0.003$ arc sec. For $\Delta \alpha_{\text {lens }}$ $=0.004$ arc sec, the deflected beam will hit the focal plane at a distance of $2.5 \mu \mathrm{m}$ from the focal point. However, at a distance of $300 \mu \mathrm{m}$ from the optical axis, a deviation $\Delta \alpha_{\text {lens }}$ of approximately 0.13 arc sec was determined. In this case, the deflected beam will hit the focal plane at a distance of $81 \mu \mathrm{m}$ from the focal point. This is not acceptable for imaging and focusing applications. The lens, which had a defect, was chosen for demonstration purposes.

The measured total phase shift $\phi_{\text {lens, } m}$ was derived from the measurement data for an effective energy of $E_{\text {eff }}=21 \mathrm{keV}$. The comparison of a line plot along the $x$ axis of $\phi_{\text {lens, } m}$ to its target value $\phi_{\text {lens }, t}$ in Fig. 4(a) exhibits strong deviations of these two functions outside a central region. The difference $\Delta \phi_{\text {lens }}$ between these two functions is shown in Fig. 4(b). The total phase shift can be directly related to a material thickness. For example, for $|x| \leqslant 100 \mu \mathrm{m}$, a maximum error of the total phase shift of $\pm 0.013 \pi$ corresponding to a thickness error of $\pm 0.5 \mu \mathrm{m}$ was determined. However, at larger distances, the error significantly rises and errors in the total phase shift of more than $\pi$, corresponding to an error of the lens thickness greater than $38 \mu \mathrm{m}$, can be found.

The data displayed in Fig. 3 characterize the beam de- 

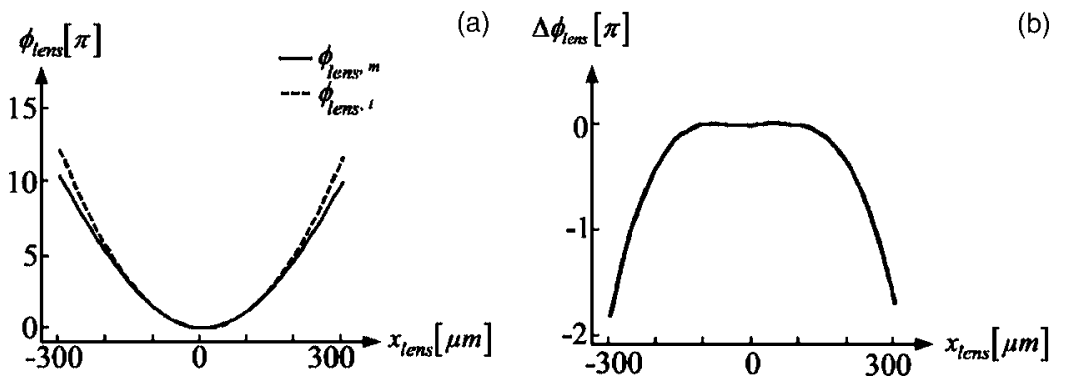

(b)

FIG. 4. Characterization of the refractive properties of a converging x-ray lens made of beryllium based on the total phase shift of $\mathrm{x}$ rays transmitted through the lens, which can be directly related to the lens thickness. The measured $\phi_{\text {lens }, m}$ (solid line) and the target behavior $\phi_{\text {lens }, t}$ (dashed line) are shown in (a) and the difference of these two functions $\Delta \phi_{\text {lens }}$ is displayed in (b).

flection angle $\alpha$ in the $x$ direction. A two-dimensional characterization of the lens profile can be achieved if orthogonal directions are measured. To obtain $\partial \phi_{\text {lens }, m} / \partial y_{\text {lens }}$, the lens was rotated by $90^{\circ}$ around the optical axis and the measurement was repeated. From the differential phase shifts in $x$ and $y$ directions, the differential phase shift in any radial direction $r$ can be derived by

$$
\frac{\partial \phi}{\partial r}=\frac{\partial \phi}{\partial x} \frac{\partial x}{\partial r}+\frac{\partial \phi}{\partial y} \frac{\partial y}{\partial r}=\frac{\partial \phi}{\partial x} \frac{x}{\sqrt{x^{2}+y^{2}}}+\frac{\partial \phi}{\partial y} \frac{y}{\sqrt{x^{2}+y^{2}}} .
$$

Figure 5 exhibits the deviation $\Delta \gamma_{\text {lens }}$ of the measured beam deflection angle $\gamma_{\text {lens }, m}$ in the radial direction from its target value $\gamma_{\text {lens, } t}$. The data was calculated using Eq. (8) and smoothed, improving noise to $\sigma_{\gamma_{\text {lens } m}} \approx 0.004$ arc sec. Apparent from the figure, $\Delta \gamma_{\text {lens }}$ is almost rotationally symmetric. The data can be interpreted in the same manner as the data presented in Fig. 3. For example, a beam transmitted through the lens inside the innermost contour line at 0.012 arc sec will hit the focal plane at a maximum distance of $7.5 \mu \mathrm{m}$ from the focal spot. In analogy, the measured beam deflection angle $\vartheta_{\text {lens, } m}$ in the angular direction can be derived. However, for the lens under investigation, no information above noise level, which is characterized by a standard deviation of $\sigma_{\vartheta_{\text {lens }, m}} \approx 0.004$ arc sec, could be found in $\vartheta_{\text {lens }, m}$.

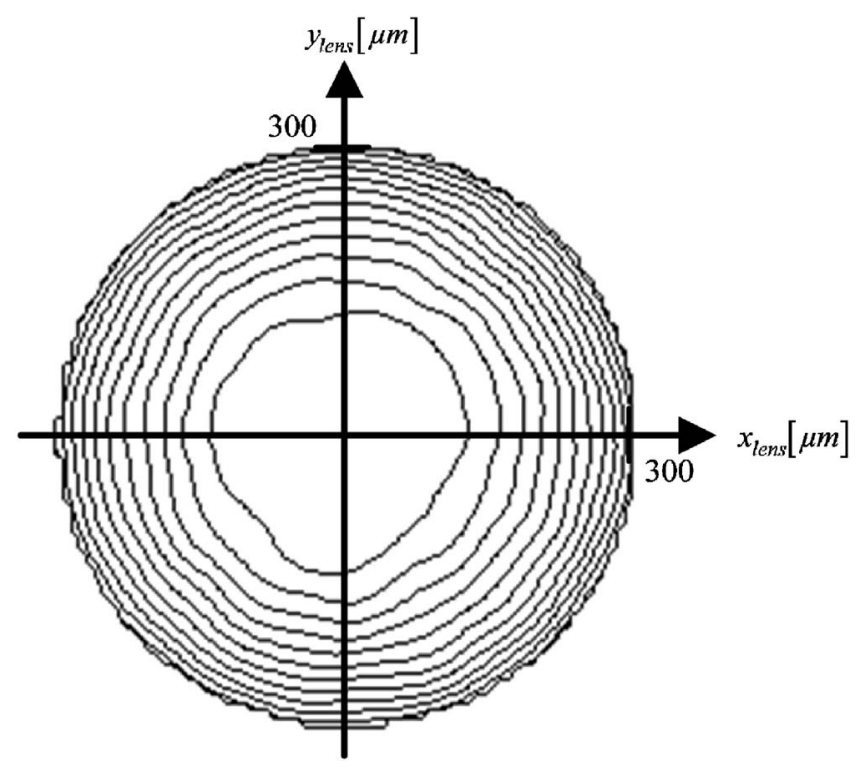

FIG. 5. Characterization of the refractive properties of a converging x-ray lens made of beryllium based on the beam deflection angle. The figure exhibits the deviation $\Delta \gamma_{\text {lens }}$ of the measured beam deflection angle in radial direction from its target value. The contour lines correspond to a deviation $\Delta \gamma_{\text {lens }}$ of $0.012,0.024,0.036$ arc sec, etc.

\section{DISCUSSION OF MEASUREMENT ERRORS DUE TO THE POLYCHROMATIC ENERGY SPECTRUM}

An error in the effective energy assumed causes a multiplicative error for the measured total phase shift $\phi_{\text {lens, } m}$, whereas the beam deflection angle $\alpha_{\text {lens, } m}$ is determined correctly. Therefore, an error in the assumed effective energy has no influence on the characterization of lens errors based on $\alpha_{\text {lens, } m \text {. }}$.

The photon energy spectrum and thereby the effective energy thereof change due to the absorption in the beam path because the x-ray attenuation factor depends on the energy. This could cause measurement errors, which affect the shape of $\alpha_{\text {lens. }}$.

Assuming a typical $\mathrm{x}$-ray spectrum for a $\mathrm{W}$ target irradiated by $50 \mathrm{keV}$ electrons, which includes the absorption of the gratings $(0.56 \mathrm{~mm} \mathrm{Si}),{ }^{12,13}$ only a minimal shift of the mean energy of the spectrum of $0.06 \mathrm{keV}$ due to the $1 \mathrm{~mm}$ thick Be lens was calculated. ${ }^{13}$ Calculations of the interference pattern shift based on a complete simulation of the interference effects using Fresnel propagation ${ }^{14}$ yield an error in the beam deflection angle determined to be less than $1 \%$ due to the spectrum shift caused by $1 \mathrm{~mm}$ Be in the beam path. These values clearly show that the lens errors determined are influenced by beam hardening only to a small extent.

\section{OUTLOOK}

A new laboratory setup for the characterization of the refractive properties of $\mathrm{X}$-ray lenses was presented. The feasibility of the method was demonstrated by a test measurement on a converging x-ray lens made of beryllium. All lens errors, which are characterized by a significant deviation of the beam deflection angle from its target value (e.g., astigmatism, coma, and spherical aberrations), can be assessed with the current setup. Systematic measurement errors due to the polychromatic energy spectrum are of minor importance for the measurement results presented. For further studies with increased accuracy or with lenses of increased attenuation (e.g., Al lenses), errors due to beam hardening could be corrected based on a measured or calculated calibration curve, which exhibits the effective energy depending on the material thickness. The spatial resolution of approximately $16 \mu \mathrm{m}$ for the measurements presented can be improved up to the focal spot size of the $\mathrm{x}$-ray source applied. Meanwhile, many microfocus $\mathrm{X}$-ray tubes with a focal spot size of $1 \mu \mathrm{m}$ or less are commercially available. 


\section{ACKNOWLEDGMENTS}

The authors would like to thank Christian Schroer and Bruno Lengeler of RWTH Aachen for supplying the lens and for very helpful discussions as well as Peter Böni and Burkhard Schillinger of Technische Universität München for fruitful discussions.

${ }^{1}$ A. Snigirev, V. Kohn, I. Snigireva, and B. Lengeler, Nature (London) 384, 49 (1996).

${ }^{2}$ B. Lengeler, C. G. Schroer, M. Kuhlmann, B. Benner, T. F. Günzler, O. Kurapova, F. Zontone, A. Snigirev, and I. Snigireva, J. Phys. D 38, A218 (2005).

${ }^{3}$ B. Lengeler, C. Schroer, J. Tümmler, B. Benner, M. Richwin, A. Snigirev, I. Snigireva, and M. Drakopoulos, J. Synchrotron Radiat. 6, 1153 (1999).

${ }^{4}$ B. Lengeler, C. G. Schroer, M. Richwin, J. Tümmler, M. Drakopoulos, A. Snigirev, and I. Snigireva, Appl. Phys. Lett. 74, 3924 (1999).

${ }_{5}^{5}$ C. G. Schroer, M. Kuhlmann, B. Lengeler, T. F. Günzler, O. Kurapova, B. Benner, C. Rau, A. S. Simionovici, A. Snigirev, and I. Snigireva, Proc. SPIE 4783, 10 (2002).

${ }^{6}$ C. David, B. Nöhammer, H. H. Solak, and E. Ziegler, Appl. Phys. Lett. 81, 3287 (2002); T. Weitkamp, B. Nöhammer, A. Diaz, C. David, and E. Ziegler, ibid. 86, 054101 (2005); A. Momose, W. Yashiro, Y. Takeda, Y.
Suzuki, and T. Hattori, Jpn. J. Appl. Phys., Part 1 45, 5254 (2006).

${ }^{7}$ F. Pfeiffer, T. Weitkamp, O. Bunk, and C. David, Nat. Phys. 2, 258 (2006).

${ }^{8}$ T. Weitkamp, A. Diaz, C. David, F. Pfeiffer, M. Stampanoni, P. Cloetens, and E. Ziegler, Opt. Express 13, 6296 (2005).

${ }^{9}$ M. Engelhardt, J. Baumann, M. Schuster, C. Kottler, F. Pfeiffer, O. Bunk, and C. David, Appl. Phys. Lett. 90, 224101 (2007).

${ }^{10}$ J. P. Guigay, S. Zabler, P. Cloetens, C. David, R. Mokso, and M. Schlenker, J. Synchrotron Radiat. 11, 476 (2004); F. Pfeiffer, O. Bunk, C. Schulze-Briese, A. Diaz, T. Weitkamp, C. David, J. F. van der Veen, I. Vartanyants, and I. K. Robinson, Phys. Rev. Lett. 94, 164801 (2005).

${ }^{11}$ Diffraction Physics, 3rd revised ed., edited by J. M. Cowley (Elsevier, Amsterdam, 1995), p. 21.

${ }^{12}$ Catalogue of X-Ray Spectra and Their Characteristic Data: ISO and DIN Radiation Qualities, Therapy and Diagnostic Radiation Qualities, Unfiltered X-Ray Spectra, edited by U. Ankerhold (Physikalisch Technische Bundesanstalt, Braunschweig, 2000), PTB Report No. PTB-Dos-34.

${ }^{13}$ J. H. Hubbell and S. M. Seltzer, Tables of X-Ray Mass Attenuation Coefficients and Mass Energy-Absorption Coefficients, National Institute of Standards and Technology (http://physics.nist.gov/PhysRefData/ XrayMassCoef/cover.html).

${ }^{14}$ M. Engelhardt, C. Kottler, O. Bunk, C. David, C. Schroer, J. Baumann, M. Schuster, and F. Pfeiffer, "The Fractional Talbot Effect in HighResolution Differential X-Ray Phase Contrast Imaging," J. Microsc. (submitted). 